\title{
Editorial comment: advances in the evaluation of liver imaging and function with gadoxetic acid-enhanced MRI
}

\author{
Christian Grieser ${ }^{1}$ (D) \\ Received: 5 April 2021 / Accepted: 12 April 2021 / Published online: 23 August 2021 \\ (C) European Society of Radiology 2021
}

For the diagnosis and staging of liver cirrhosis, biopsy is still the current reference standard; however, it is associated with limitations such as increased costs, patient acceptance, and complications as well as limited sampling area and resultant bias $[1,2]$. In recent years, efforts have been made to migrate toward non-invasive techniques for assessing liver cirrhosis and to identify patients at high risk for decompensation. Magnetic resonance imaging (MRI) of the liver with gadoxetic acid is widely used in clinical routine and has been proven to be a powerful tool in the diagnosis of focal liver disease as well as estimation of hepatic function $[3,4]$. Recently, Bastati and colleagues developed the functional liver imaging score (FLIS), a simple method for hepatic function assessment based on three features on hepatobiliary phase images - liver parenchymal enhancement, biliary contrast excretion, and portal vein sign [5]. However, up to date, no study has directly demonstrated the relationship between FLIS and parameters representing hepatic function. This association between FLIS and first hepatic decompensation was evaluated in a study by Lee et al [6] which is published in this journal. A total of 134 patients which were diagnosed with liver cirrhosis or chronic liver disease and underwent gadoxetic acid-enhanced MRI were retrospectively included. The authors were able to show how a simple visual appreciation of the hepatobiliary phase allows a reliable stratification of patients, very similar to Child Pugh score. Therefore, three parameters on hepatobiliary phase images were evaluated for FLIS: liver parenchymal enhancement as compared with the kidney (less, similar, or higher), biliary excretion enhancement (non, mean, high), and portal vein sign (portal vein contrast less, same or with higher intensity as compared with the liver). This score leads to a 6-point score that showed a strong to very

This comment refers to the articles available at https://doi.org/10.1007/ s00330-021-07805-0 and https://doi.org/10.1007/s00330-021-07955-1.

Christian Grieser

christian.grieser@zemodi.de

1 ZEMODI - Zentrum für moderne Diagnostik, Schwachhauser Heerstrasse 63a, 28211 Bremen, Germany strong correlation with Child-Pugh score ( $r=-0.60$ to 0.82$)$. ROC curve analysis showed that FLIS $>5$ was the optimal cut-off for prediction of Child-Pugh score class A or chronic liver disease (sensitivity, $83.7 \%$; specificity, $94.4 \%$; area under the curve [AUC], 0.93). FLIS $<5$ was independently associated with the development of first hepatic decompensation in patients with Child-Pugh score class A. The authors conclude that FLIS shows a strong correlation with hepatic function and can stratify the chronic liver disease class. In addition, FLIS can help prediction for the development of first liver decompensation which is important in the clinical setting and validation.

Furthermore, other MRI techniques, such as T1 and T2 mapping, are present today and have already shown promising results for predicting decompensated cirrhosis: Prolonged T1 values due to the decrease of hepatic parenchymal enhancement in the hepatobiliary phase with gadoxetic acid and similarly, liver T2 values have been reported to be prolonged due to remodeling during fibrosis progression [7-9]. However, for daily use in a clinical setting, conventional MR sequences are known to be limited because of their long acquisition time and motion sensitivity. Recently, new fast radial turbo spin-echo sequences were introduced resulting in fast, high-resolution T2 mapping of the liver. However, the usefulness of T2 mapping for predicting liver-related events has not yet been evaluated clinically. Published in this issue, a study by Yang et al [10] showed that this fast T2Liver from T2 mapping can predict decompensation and death in patients with liver cirrhosis. Yang et al included 292 cirrhotic patients who underwent gadoxetic acid-enhanced MRI in this retrospective study. T1 and $\mathrm{T} 2$ values of the liver and spleen were measured and the association of MR parameters and serum markers with decompensation and death were investigated. A prolonged T2Liver was independently predictive of decompensation along with the serum albumin level and Model for EndStage Liver Disease (MELD) score. T2Liver and serum albumin level were independent predictors of death. The authors state that T2 mapping should be recommended as part of liver MRI examinations for cirrhotic patients because it can provide 
a non-invasive prognostic marker for the development of decompensation and death.

To conclude, MRI with the use of gadoxetic acid is a quite powerful non-invasive tool for the evaluation of liver function and predicting decompensation and death in patients with cirrhosis. New, rather easy methods like the presented FLIS and furthermore more complex technics like T2 mapping should be taken into consideration and may be implemented in the abdominal MRI protocols to identify patients at high risk for decompensation. However, more prospective studies using larger multicentre samples with various etiologies of cirrhosis and MRI settings are needed to address its current limitations and to evaluate the overall clinical impact.

Funding The author states that this work has not received any funding.

\section{Declarations}

Guarantor The scientific guarantor of this publication is Christian Grieser.

Conflict of interest The author of this manuscript declares no relationships with any companies, whose products or services may be related to the subject matter of the article.

Statistics and biometry No complex statistical methods were necessary for this paper.

Informed consent Written informed consent was not required for this study because this is an editorial without any study subjects.

Ethical approval Institutional Review Board approval was not required because this is an editorial without any study subjects.

\section{Methodology}

- Editorial comment

\section{References}

1. Rockey DC, Caldwell SH, Goodman ZD et al (2009) Liver biopsy. Hepatology 49:1017-1044

2. Bravo AA, Sheth SG, Chopra S (2001) Liver biopsy. N Engl J Med 344:495-500

3. Yamada A, Hara T, Li F et al (2011) Quantitative evaluation of liver function with use of gadoxetate disodium-enhanced MR imaging. Radiology 260:727-733

4. Verloh N, Haimerl M, Zeman F et al (2014) Assessing liver function by liver enhancement during the hepatobiliary phase with GdEOB-DTPA-enhanced MRI at 3 Tesla. Eur Radiol 24:1013-1019

5. Bastati N, Wibmer A, Tamandl D et al (2016) Assessment of orthotopic liver transplant graft survival on gadoxetic acidenhanced magnetic resonance imaging using qualitative and quantitative parameters. Invest Radiol 51:728-734

6. Lee HJ, Hong SB, Lee NK et al (2021) Validation of functional liver imaging scores (FLIS) derived from gadoxetic acid- enhanced mri in patients with chronic liver disease and liver cirrhosis: the relationship between Child-Pugh score and FLIS. Eur Radiol. https://doi.org/10.1007/s00330-021-07955-1

7. Heye T, Yang SR, Bock M et al (2012) MR relaxometry of the liver: significant elevation of $\mathrm{T} 1$ relaxation time in patients with liver cirrhosis. Eur Radiol 22:1224-1232

8. Cassinotto C, Feldis M, Vergniol J et al (2015) MR relaxometry in chronic liver diseases: Comparison of T1 mapping, T2 mapping, and diffusion-weighted imaging for assessing cirrhosis diagnosis and severity. Eur J Radiol 84:1459-1465

9. Luetkens JA, Klein S, Traeber F et al (2018) Quantitative liver MRI including extracellular volume fraction for non-invasive quantification of liver fibrosis: a prospective proof-of-concept study. Gut 67: 593-594

10. Yang W, Kim JE, Choi HC et al (2021) T2 mapping in gadoxetic acidenhanced MRT: utility for predicting decompensation and death in cirrhosis. Eur Radiol. https://doi.org/10.1007/s00330021-07805-0

Publisher's note Springer Nature remains neutral with regard to jurisdictional claims in published maps and institutional affiliations. 